\title{
Set of sum rules for anomalous gauge boson couplings
}

\author{
Joannis Papavassiliou* \\ Theory Division, CERN, CH-1211 Geneva 23, Switzerland
}

Kostas Philippides ${ }^{\dagger}$

Department of Physics, University of Durham, Durham, DH1 3LE, United Kingdom

(Received 7 July 1998; revised manuscript received 9 October 1998; published 8 February 1999)

\begin{abstract}
The dependence of the differential cross section for on-shell $W$-pair production on the anomalous trilinear gauge couplings invariant under $C$ and $P$ is examined. It is shown that the contributions of the anomalous magnetic moments of the $W$ boson due to the photon and the $Z$ can be individually projected out by means of two appropriately constructed polynomials. The remaining four anomalous couplings are shown to satisfy a set of model-independent sum rules. Specific models which predict special relations among the anomalous couplings are then studied, in particular, the composite model of Brodsky and Hiller and linear and non-linear effective Lagrangian approaches. The relations predicted by these models, when combined with the aforementioned sum rules, give rise to definite predictions, particular to each model. These predictions can be used, at least in principle, in order to exclude or constrain such models. Finally, we present an elementary discussion of the statistical properties of the proposed observables.
\end{abstract}

[S0556-2821(99)04505-1]

PACS number(s): 14.70.Fm, 11.55.Hx, 12.60.Rc, 13.40.Gp

\section{INTRODUCTION}

The possibility of probing directly non-Abelian vertices in the experiments at the CERN Large Electron Positron collider LEP2 through the process $e^{+} e^{-} \rightarrow W^{+} W^{-}[1-3]$, as well as the Fermilab Tevatron [4] and the Next Linear Collider (NLC), has motivated extensive study of anomalous gauge boson couplings. The general methodology for quantifying the effects of such couplings on physical amplitudes was presented some time ago in the classic papers of Gaemers and Gounaris [5] and Hagiwara et al. [6]. The central idea is to parametrize the most general three-gauge-boson vertex allowed by Lorentz invariance in terms of unknown form factors, compute the theoretical predictions of relevant physical amplitudes using this vertex, and then attempt to extract information about the structure of these form factors by comparing these theoretical predictions with the experimental data. In practice one usually obtains experimental lower bounds on the size of these form factors by carrying out a multi-parameter fit to the data [7]. This type of analysis becomes considerably more complicated if one takes into account the fact that the two produced $W$ are not stable, but decay subsequently through a variety of channels. The complexity of this problem necessitates a detailed amplitude analysis for the process $e^{+} e^{-} \rightarrow W^{+} W^{-}$; several studies based on a variety of methods, such as helicity amplitude techniques [8], have been carried out, and a plethora of complementary observables, such as integrated cross sections, angular distributions, polarized cross sections, $W$ density matrices, and polarization asymmetries, have been proposed [9].

It would clearly be useful to relate directly some of the

\footnotetext{
*Email address: Joannis.Papavassiliou@ cern.ch

†Email address: Kostas.Philippides@durham.ac.uk
}

anomalous form factors to experimentally measurable quantities. In addition, it is important to establish a variety of ways for testing experimentally some of the characteristic predictions of models which give rise to such anomalous form factors. To that end in this paper we present a study complementary to that of Ref. [6]. In particular, we compute the differential cross section for the process $e^{+} e^{-}$ $\rightarrow W^{+} W^{-}$, under the assumptions that (i) the anomalous form factors used to parametrize the non-Abelian vertex satisfy separately the discrete symmetries $C, P$, and $T$. This assumption, which is often employed in the literature, reduces the number of possible form factors from 14 down to 6. (ii) The size of the anomalous couplings is small compared to unity, so that we may keep only effects linear in them. And (iii) the two $W$ 's are strictly on shell; i.e., we do not consider the effect of their subsequent decays. Then, following a method developed in Ref. [10], a system of four independent algebraic equations for the six unknown anomalous form factors is derived. It turns out that one can solve directly two of these equations and obtain explicit expressions for the two form factors traditionally associated with the photon and $Z$ anomalous magnetic moments of the $W$ in terms of the differential cross section. These two expressions are completely model independent; therefore they can serve as a testing ground for confronting the predictions of different models for the anomalous magnetic moments with experiment. The two remaining equations constitute a set of model-independent sum rules [11] for the other four unknown form factors.

To demonstrate with specific examples the potential usefulness of such sum rules for testing the viability of models predicting anomalous couplings, or at least for constraining them, we first study the composite model proposed by Brodsky and Hiller (BH) [12]. This model predicts certain relations between the anomalous form factors; if these relations are fed into the two remaining equations mentioned above, 
the system turns out to be over-constrained, thus leading to two independent predictions. These predictions are particular to this composite model, and could, at least in principle, be confronted with experiment. Next we turn to an approach based on a gauge invariant, effective Lagrangian [13-17], which also provides relations among the various anomalous form factors; in particular all form factors are expressed in terms of the three free parameters of the effective Lagrangian. When these relations are combined with the two aforementioned equations as before, one constraint emerges, which constitutes a particular prediction of this effective Lagrangian approach.

The paper is organized as follows: In Sec. II we briefly review the general form of the three-gauge-boson vertex and define the anomalous form factors which parametrize the deviation of the couplings from the standard model (SM) treelevel values. In Sec. III we compute under the three assumptions mentioned above, the analytic dependence of the theoretical cross section on these form factors, and show how to derive expressions for the anomalous magnetic moments, as well as the two sum rules. In Sec. IV we study some aspects of the $\mathrm{BH}$ model, and translate its predictions at the level of experimental cross sections. In Sec. V the effective Lagrangian approach is outlined both for a linear and nonlinear realizations of the symmetry and its predictions are expressed in terms of experimental cross sections. In Sec. VI we present an elementary discussion of the statistical properties of the proposed observables. Finally in Sec. VII we summarize our results.

\section{ANOMALOUS COUPLINGS}

In this section we give a brief review of the anomalous gauge-boson couplings and establish notation. The SM threegauge-boson vertex $V_{\mu} W_{\alpha}^{-} W_{\beta}^{+}$involving a neutral gauge boson $V=\gamma, Z$ coupled to a conserved current (massless external fermions in the case of the $Z$ boson) and two on-shell $W$ 's is given by [18]

$$
\Gamma_{\mu \alpha \beta}^{V, 0}\left(q,-p_{1},-p_{2}\right)=g_{V} \Gamma_{\mu \alpha \beta}^{0}\left(q,-p_{1},-p_{2}\right),
$$

with

$$
\Gamma_{\mu \alpha \beta}^{0}\left(q,-p_{1},-p_{2}\right)=\left(p_{2}-p_{1}\right)_{\mu} g_{\alpha \beta}+2\left(q_{\beta} g_{\mu \alpha}-q_{\alpha} g_{\beta \mu}\right),
$$

where $g_{\gamma}=g s_{w}, g_{Z}=g c_{w}, g$ is the $S U(2)_{L}$ gauge coupling, and $s_{w}^{2}=1-c_{w}^{2}$ is sine of the weak mixing angle. $\Gamma_{\mu \alpha \beta}^{0}$ satisfies the following elementary Ward identity:

$$
q^{\mu} \Gamma_{\mu \alpha \beta}^{0}\left(q,-p_{1},-p_{2}\right)=\left[p_{2}^{2}-p_{1}^{2}\right] g_{\alpha \beta} .
$$

Assuming that the two $W$ 's are on shell, i.e., $p_{1}^{2}=p_{2}^{2}=M_{W}^{2}$, we have from Eq. (2.3) that

$$
q^{\mu} \Gamma_{\mu \alpha \beta}^{0}\left(q,-p_{1},-p_{2}\right)=0 .
$$

The above elementary Ward identities are crucial for the gauge invariance of the process $f^{+} f^{-} \rightarrow W^{+} W^{-}$even if the external fermionic current is conserved. To appreciate this fact all one has to do is to choose to work in the axial or planar gauge [19] instead of the usual renormalizable $\left(R_{\xi}\right)$ gauges [20]. In that case, the bare photon propagator $\Delta_{0}^{\mu \nu}(q)$ appearing in graph 1(a) assumes the form

$$
\Delta_{0}^{\mu \nu}(q)=\left[g^{\mu \nu}-\frac{\eta^{\mu} q^{\nu}+\eta^{\nu} q^{\mu}}{\eta q}\right] \frac{1}{q^{2}}+\eta^{2} \frac{q^{\mu} q^{\nu}}{(\eta q)^{2}} .
$$

The four-vector $\eta_{\mu}$ in the above expressions is a gaugefixing parameter; therefore, physical quantities such as $S$-matrix elements should be independent of $\eta_{\mu}$. Evidently, even though all $\eta$-dependent terms proportional to $q^{\mu}$ and $q^{\mu} q^{\nu}$ will vanish when contracted with the conserved current, the $\eta$-dependent term proportional to $q^{\nu}$ can only cancel if the Ward identity of Eq. (2.4) holds.

The most general parametrization of the trilinear gauge vertex consistent with Lorentz invariance is given in terms of 14 form factors $f_{i}^{V}[5,6]$ :

$$
\begin{aligned}
\Gamma_{\mu \alpha \beta}^{V} & \left(q,-p_{1},-p_{2}\right) \\
= & \left(1+f_{1}^{V}\right)\left(p_{2}-p_{1}\right)_{\mu} g_{\alpha \beta}+2\left(1+f_{3}^{V}\right)\left(q_{\beta} g_{\mu \alpha}-q_{\alpha} g_{\beta \mu}\right) \\
& -f_{2}^{V} q_{\alpha} q_{\beta}\left(p_{2}-p_{1}\right)_{\mu} / 2 M_{W}^{2}-i f_{4}^{V}\left(q_{\beta} g_{\mu \alpha}+q_{\alpha} g_{\beta \mu}\right) \\
& +i f_{5}^{V} \epsilon^{\mu \alpha \beta \rho}\left(p_{2}-p_{1}\right)_{\rho} \\
& +f_{6}^{V} \epsilon^{\mu \alpha \beta \rho} q_{\rho}+f_{7}^{V}\left(p_{2}-p_{1}\right)_{\mu} \epsilon^{\alpha \beta \rho \sigma} q_{\rho}\left(p_{2}-p_{1}\right)_{\sigma} / M_{W}^{2} .
\end{aligned}
$$

The first three form factors $f_{i}, i=1,2,3$, preserve $C$ and $P$ separately. $f_{5}$ respects $C P$ but violates both $C$ and $P$. The rest of the form factors violate $C P: f_{4}$ is $P$ even but $C$ odd, while $f_{6}$ and $f_{7}$ are $C$ even and $P$ odd. Note the slight difference in notation compared to Ref. [6]; we have chosen to write the vertex in a way such that the form factors $f_{i}^{V}$ express exactly the deviations of the couplings from their SM tree level values. Indeed, by comparing Eq. (2.6) with Eq. (2.2) we see that all form factors $f_{i}^{V}$ are normalized to be zero at the tree level. The above form factors receive nonzero contributions of order $O(\alpha)$ from one-loop quantum corrections within the SM [21,22]; in fact, $f_{4}^{V}, f_{5}^{V}, f_{6}^{V}$, and $f_{7}^{V}$ receive contributions from one-loop fermionic diagrams only [6]. The contributions to the $f_{i}^{V}$ obtained from supersymmetric [23] and other extensions of the SM [24], as well as composite models [25], have been studied extensively in the recent literature. In what follows we will treat the $f_{i}^{V}$ as if they were small with respect to unity, but not necessarily of order $\mathcal{O}(\alpha)$.

It is possible to impose constraints on the form of the $f_{i}^{V}$ by resorting to various physical and field-theoretical considerations. For example, if one requires that $\Gamma_{\mu \alpha \beta}^{\gamma}$ satisfy the Ward identity of Eq. (2.4), namely $q^{\mu} \Gamma_{\mu \alpha \beta}^{\gamma}=0$, in order for the cancellation of the gauge-dependent terms stemming from the tree-level photon propagator of Eq. (2.5) to go through as before, the $f_{4}$ and $f_{5}$ terms in the $\gamma W^{+} W^{-}$vertex $\Gamma_{\mu \alpha \beta}^{\gamma}$ need to be replaced by 


$$
\begin{aligned}
& -i f_{4}^{\gamma}\left[q_{\beta} g_{\mu \alpha}+q_{\alpha} g_{\beta \mu}-2 q^{\mu} q^{\alpha} q^{\beta} / q^{2}\right]+i f_{5}^{\gamma}\left[\epsilon^{\mu \alpha \beta \rho}\left(p_{2}-p_{1}\right)_{\rho}\right. \\
& \left.-q^{\mu} \epsilon^{\alpha \beta \rho \sigma} q^{\rho}\left(p_{2}-p_{1}\right)_{\sigma} / q^{2}\right] .
\end{aligned}
$$

Analyticity at $q^{2}=0$ provides us then with the constraint $f_{4}^{\gamma}\left(q^{2}=0\right)=f_{5}^{\gamma}\left(q^{2}=0\right)=0$. In addition, fixing the electric charge of the $W^{ \pm}$to be \pm 1 imposes the additional constraint $f_{1}^{\gamma}\left(q^{2}=0\right)=0$.

If the form factors $f_{i}^{V}$ are kept arbitrary, the vertex of Eq. (2.6) leads to cross sections which grossly violate unitarity, because the subtle cancellations enforced by the tree-level couplings of the SM are now distorted [26,27]. Unitarity can only be restored if the form factors $f_{i}\left(q^{2}\right)$ fall sufficiently fast with increasing $q^{2}$. In fact in analyzing the effects of anomalous couplings in hadron colliders that probe a wide range of $q^{2}$ a behavior of the following form is assumed:

$$
f_{i}\left(q^{2}\right)=\frac{f_{i}^{0}}{\left(1+q^{2} / \Lambda^{2}\right)^{2}} .
$$

The exact form of the form factors depends on the underlying dynamics that generate them and determine the scale $\Lambda$. Tree level unitarity then provides order of magnitude estimates for the product $f_{i}^{0} \Lambda[28,31]$.

\section{W PAIR PRODUCTION WITH ANOMALOUS FORM FACTORS}

We now proceed to calculate the process

$$
e^{-}\left(k_{1}, s_{1}\right) e^{+}\left(k_{2}, s_{2}\right) \rightarrow W^{-}\left(p_{1}, \lambda_{1}\right) W^{+}\left(p_{2}, \lambda_{2}\right)
$$

using the non-standard vertex of Eq. (2.6) and keeping only terms linear in the anomalous couplings $f_{i}^{V}$. For the rest of this paper we restrict ourselves only to the form factors that separately respect the discrete $C, P$, and $T$ symmetries. The electrons are considered massless, and $s_{i}, \lambda_{i}$ label respectively the initial electron's and positron's spins and the polarizations of the final $W$ 's. The relevant kinematical variables in the center-of-mass frame are

$$
\begin{aligned}
& s=\left(k_{1}+k_{2}\right)^{2}=\left(p_{1}+p_{2}\right)^{2}, \\
& t=\left(k_{1}-p_{1}\right)^{2}=\left(p_{2}-k_{2}\right)^{2}=-\frac{s}{4}\left(1+\beta^{2}-2 \beta \cos \theta\right),
\end{aligned}
$$

where

$$
\beta=\sqrt{1-\frac{4 M_{W}^{2}}{s}}
$$

is the velocity of the $W^{\prime}$ s, and $\theta$ is the angle between the incoming electron and the outgoing $W^{-}$.

The $S$-matrix element for this process is given by

$$
\begin{aligned}
& i\left\langle W^{+} W^{-}|T| e^{+} e^{-}\right\rangle \\
& \quad=i \epsilon_{\alpha}^{*}\left(p_{1}, \lambda_{1}\right) \epsilon_{\beta}^{*}\left(p_{2}, \lambda_{2}\right) \bar{v}\left(k_{2}, s_{2}\right) T^{\alpha \beta} u\left(k_{1}, s_{1}\right),
\end{aligned}
$$

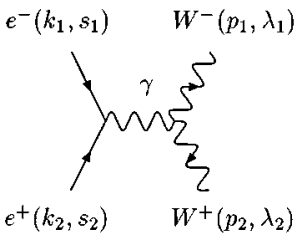

(a)

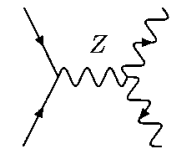

(b)

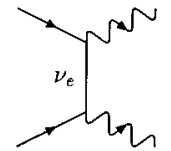

(c)
FIG. 1. The three diagrams which contribute to the process $e^{+} e^{-} \rightarrow W^{+} W^{-}$at the tree level in the case of massless electrons.

where the amplitude $T^{\alpha \beta}$ is the sum of the graphs of Fig. 1. If we now write the vertex of Eq. (2.6) as a sum of the standard vertex of Eq. (2.2) and a non-standard piece, i.e.,

$$
\begin{aligned}
\Gamma_{\mu \alpha \beta}^{V}\left(q,-p_{1},-p_{2}\right) \equiv & \Gamma_{\mu \alpha \beta}^{V, 0}\left(q,-p_{1},-p_{2}\right) \\
& +\delta \Gamma_{\mu \alpha \beta}^{V}\left(q,-p_{1},-p_{2}\right),
\end{aligned}
$$

then $T^{\alpha \beta}$ consists of the standard part $T_{\alpha \beta}^{0}$ and a nonstandard part $\delta T_{\alpha \beta}$, which originates from the $s$-channel graphs only, i.e.,

$$
T_{\alpha \beta}=T_{\alpha \beta}^{0}+\delta T_{\alpha \beta} .
$$

The two terms are given explicitly by

$$
\begin{aligned}
T_{\alpha \beta}^{0}= & i g^{2}\left[s_{w}^{2} \gamma^{\mu} \frac{1}{s}+\gamma^{\mu}\left(v-a \gamma_{5}\right) \frac{1}{s-M_{Z}^{2}}\right] \Gamma_{\mu \alpha \beta}^{0} \\
& -i \frac{g^{2}}{2} \gamma_{\beta} P_{L} \frac{1}{k_{1}-p_{1}} \gamma_{\alpha} P_{L}
\end{aligned}
$$

and

$$
\delta T_{\alpha \beta}=i g^{2} s_{w}^{2} \gamma^{\mu} \frac{1}{s} \delta \Gamma_{\mu \alpha \beta}^{\gamma}+\gamma^{\mu}\left(v-a \gamma_{5}\right) \frac{1}{s-M_{Z}^{2}} \delta \Gamma_{\mu \alpha \beta}^{Z} .
$$

In the above equations $P_{L}=\left(1-\gamma_{5}\right) / 2$ is the left chirality projector and $v=1 / 4-s_{w}^{2}, a=1 / 4$ are respectively the vector and axial couplings of the electron with the $Z$.

Defining the $W$ polarization tensor as $Q_{\mu \nu}(k)$ $\equiv \sum \epsilon_{\mu}(k) \epsilon_{\nu}^{*}(k)=-g_{\mu \nu}+k_{\mu} k_{\nu} / M_{W}^{2}$, the modulus squared of the matrix element averaged over initial state spins, summed over the final polarizations, and to first order in the deviations is given by

$$
\begin{aligned}
& \sum_{s_{1}, s_{2}} \sum_{\lambda_{1}, \lambda_{2}}\left|\left\langle e^{+} e^{-}|T| W^{+} W^{-}\right\rangle\right|^{2} \\
& =\sum_{s_{1}, s_{2}}\left(\bar{v} T_{\mu^{\prime} \nu^{\prime}} u\right)^{*} Q^{\mu^{\prime} \mu}\left(p_{1}\right) Q^{\nu^{\prime} \nu}\left(p_{2}\right)\left(\bar{v} T_{\mu \nu} u\right) \\
& =\sum_{s_{1}, s_{2}}\left(\bar{v} T_{\mu^{\prime} \nu^{\prime}}^{0} u\right)^{*} Q^{\mu^{\prime} \mu}\left(p_{1}\right) Q^{\nu^{\prime} \nu}\left(p_{2}\right)\left(\bar{v} T_{\mu \nu}^{0} u\right) \\
& \quad+2 \sum_{s_{1}, s_{2}} \operatorname{Re}\left[\left(\bar{v} T_{\mu^{\prime} \nu^{\prime}}^{0} u\right)^{*} Q^{\mu^{\prime} \mu}\left(p_{1}\right) Q^{\nu^{\prime} \nu}\left(p_{2}\right)\right. \\
& \left.\quad \times\left(\bar{v} \delta T_{\mu \nu} u\right)\right] .
\end{aligned}
$$


The unpolarized differential cross section in the center-ofmass frame is given by

$$
\frac{d \sigma}{d \Omega}=\frac{1}{256 \pi^{2}} \frac{\beta}{s} \sum_{s_{1}, s_{2}} \sum_{\lambda_{1}, \lambda_{2}}\left|\left\langle e^{+} e^{-}|T| W^{+} W^{-}\right\rangle\right|^{2},
$$

and consists of a standard and an anomalous contribution

$$
\frac{d \sigma}{d \Omega}=\frac{d \sigma^{0}}{d \Omega}+\frac{d \sigma^{a n}}{d \Omega},
$$

given by

$$
\begin{aligned}
\frac{d \sigma^{0}}{d \Omega}= & \frac{1}{256 \pi^{2}} \frac{\beta}{s} \sum_{s_{1}, s_{2}}\left(\bar{v} T_{\mu^{\prime} \nu^{\prime}}^{0} u\right)^{*} Q^{\mu^{\prime} \mu}\left(p_{1}\right) Q^{\nu^{\prime} \nu}\left(p_{2}\right) \\
& \times\left(\bar{v} T_{\mu \nu}^{0} u\right)
\end{aligned}
$$

and

$$
\begin{aligned}
\frac{d \sigma^{a n}}{d \Omega}= & \frac{1}{128 \pi^{2}} \frac{\beta}{s} \sum_{s_{1}, s_{2}} \operatorname{Re}\left[\left(\bar{v} T_{\mu^{\prime} \nu^{\prime}}^{0} u\right)^{*} Q^{\mu^{\prime} \mu}\left(p_{1}\right) Q^{\nu^{\prime} \nu}\left(p_{2}\right)\right. \\
& \left.\times\left(\bar{v} \delta T_{\mu \nu} u\right)\right],
\end{aligned}
$$

respectively. The SM cross section $d \sigma^{0} / d \Omega$ in Eq. (3.12) has been calculated in Ref. [29]; we do not report it here. We next turn to the computation of the non-standard part of the cross section $d \sigma^{a n} / d \Omega$. We define the variables

$$
x=\cos \theta, \quad z=\frac{1+\beta^{2}}{2 \beta},
$$

in terms of which the Mandelstam variable $t$, defined in Eq. (3.2), becomes

$$
t=-\frac{s}{4}\left(1+\beta^{2}-2 \beta x\right)=-\frac{s \beta}{2}(z-x) .
$$

Then, a straightforward calculation yields

$$
(z-x) \frac{d \sigma^{a n}}{d \Omega}=\frac{1}{128 \pi^{2}} g^{4} \frac{\beta}{s} \sum_{i=1}^{4} \sigma_{i}(s) P_{i}(x, s) .
$$

The functions $P_{i}(x, s)$ are polynomials in $x$ of maximum degree 3 , given by

$$
\begin{aligned}
& P_{1}(x, s)=z-x, \\
& P_{2}(x, s)=(z-x)\left(1-x^{2}\right), \\
& P_{3}(x, s)=1-x^{2}, \\
& P_{4}(x, s)=1-\beta x .
\end{aligned}
$$

In Eq. (3.16) the terms proportional to $P_{1}$ and $P_{2}$ originate from the square of the $s$-channel graphs, whereas the terms proportional to $P_{3}$ and $P_{4}$ from the interference be- tween the $s$-channel graphs and the $t$-channel graph. Notice that there is no contribution to $d \sigma^{a n} / d \Omega$ originating from the square of the $t$-channel graph; this is so because all such contributions are absorbed into the standard part, since the couplings of the neutral gauge bosons to fermions are assumed to be exactly those of the SM.

The functions $\sigma_{i}(s)$ are linear combinations of the various deviation form factors. Specifically,

$$
\begin{aligned}
& \sigma_{1}(s)=A_{1} f_{3}^{\gamma}+A_{2} f_{3}^{Z}, \\
& \sigma_{2}(s)=A_{3} f_{1}^{\gamma}+A_{4} f_{1}^{Z}+A_{5} f_{3}^{\gamma}+A_{6} f_{3}^{Z}+A_{7} f_{2}^{\gamma}+A_{8} f_{2}^{Z}, \\
& \sigma_{3}(s)=A_{9} f_{1}^{\gamma}+A_{10} f_{1}^{Z}-\eta A_{9} f_{2}^{\gamma}-\eta A_{10} f_{2}^{Z}, \\
& \sigma_{4}(s)=A_{11} f_{3}^{\gamma}+A_{12} f_{3}^{Z},
\end{aligned}
$$

where $\eta=s / 4 M_{W}^{2}$. The explicit closed expressions for the coefficients $A_{i}$ are given below. Setting

$$
\begin{aligned}
& y=\frac{s}{s-M_{Z}^{2}} \frac{1}{c_{w}^{2}}, \\
& r=a^{2}+v^{2},
\end{aligned}
$$

we have

$$
\begin{aligned}
& A_{1}=-8 s_{w}^{2}\left[y v\left(4 c_{w}^{2}-1\right)+4 s_{w}^{2}\right] \\
& A_{2}=-8 y c_{w}^{2}\left[y r\left(4 c_{w}^{2}-1\right)+4 s_{w}^{2} v\right] \\
& A_{3}=\beta^{2} s_{w}^{2}\left[2(3-2 \eta)\left(y v c_{w}^{2}+s_{w}^{2}\right)-(1+2 \eta) y v\right] \\
& A_{4}=\beta^{2} y c_{w}^{2}\left[2(3-2 \eta)\left(y r c_{w}^{2}+v s_{w}^{2}\right)-(1+2 \eta) y r\right] \\
& A_{5}=-4 \beta^{2} \eta s_{w}^{2}\left[y v\left(2 c_{w}^{2}-1\right)+2 s_{w}^{2}\right] \\
& A_{6}=-4 \beta^{2} \eta y c_{w}^{2}\left[y r\left(2 c_{w}^{2}-1\right)+2 v s_{w}^{2}\right] \\
& A_{7}=-2 \beta^{2} \eta s_{w}^{2}\left[2(1+\eta)\left(y v c_{w}^{2}+s_{w}^{2}\right)-\eta y v\right], \\
& A_{8}=-2 \beta^{2} \eta y c_{w}^{2}\left[2(1+\eta)\left(y r c_{w}^{2}+v s_{w}^{2}\right)-\eta y r\right] \\
& A_{9}=-\beta s_{w}^{2} \\
& A_{10}=-(a+v) \beta y c_{w}^{2} \\
& A_{11}=4 s_{w}^{2} \beta^{-1} \\
& A_{12}=4 y(a+v) c_{w}^{2} \beta^{-1} .
\end{aligned}
$$

The values of the coefficients $A_{i}(s)$ for some typical LEP2 energies are shown in Table I. Notice that the coefficients $A_{i}$ appearing within each of the four equations in Eqs. (3.18) are of the same order of magnitude, and therefore none of them can be neglected. 
TABLE I. The coefficients $A_{i}$ as a function of $\sqrt{s}$.

\begin{tabular}{cccccc}
\hline \hline$\sqrt{s}(\mathrm{GeV})$ & 161 & 172 & 180 & 192 & 200 \\
\hline$A_{1}$ & -1.79 & -1.78 & -1.77 & -1.77 & -1.76 \\
$A_{2}$ & -3.25 & -2.91 & -2.73 & -2.53 & -2.42 \\
$A_{3}$ & $2.20 \times 10^{-3}$ & $7.40 \times 10^{-2}$ & 0.123 & 0.191 & 0.235 \\
$A_{4}$ & $3.68 \times 10^{-3}$ & $11.0 \times 10^{-2}$ & 0.169 & 0.239 & 0.279 \\
$A_{5}$ & $-1.70 \times 10^{-3}$ & $-6.18 \times 10^{-2}$ & -0.108 & -0.181 & -0.232 \\
$A_{6}$ & $-1.83 \times 10^{-3}$ & $-6.03 \times 10^{-2}$ & -0.0991 & -0.154 & -0.190 \\
$A_{7}$ & $-1.79 \times 10^{-3}$ & $-6.94 \times 10^{-2}$ & -0.127 & -0.228 & -0.306 \\
$A_{8}$ & $-3.24 \times 10^{-3}$ & $-11.1 \times 10^{-2}$ & -0.187 & -0.303 & -0.385 \\
$A_{9}$ & $-1.41 \times 10^{-2}$ & $-7.98 \times 10^{-2}$ & -0.101 & -0.122 & -0.133 \\
$A_{10}$ & $-2.56 \times 10^{-2}$ & $-13.7 \times 10^{-2}$ & -0.167 & -0.195 & -0.208 \\
$A_{11}$ & 14.2 & 2.51 & 1.99 & 1.64 & 1.50 \\
$A_{12}$ & 25.8 & 4.31 & 3.30 & 2.61 & 2.34 \\
\hline \hline
\end{tabular}

The polynomials $P_{i}(x)$ are linearly independent; indeed, their Wronskian is given by

$$
W\left(P_{i}\right)=\frac{24 M_{W}^{2}}{s},
$$

which can be zero only if $s \rightarrow \infty$.

It is important to emphasize at this point that in our case the quantity $(z-x)\left(d \sigma^{a n} / d \Omega\right)$ appearing on the right hand side of Eq. (3.16) is more suitable as an experimental observable than $\left(d \sigma^{a n} / d \Omega\right)$ itself [10]. The reason is that this quantity is the sum of a finite number of linearly independent polynomials. Instead, an expansion of $\left(d \sigma^{a n} / d \Omega\right)$ in terms of polynomials in $x$ would necessitate an infinite number of them, because of the presence of the term $(z-x)^{-1}$. This fact would in turn complicate the inversion of such a relation, i.e., the determination of the quantities $\sigma_{i}$, which contain the dependence on the $f_{i}^{V}$. We next proceed to carry this inversion for the quantity $(z-x)\left(d \sigma^{a n} / d \Omega\right)$.

To accomplish this one must construct a set of four other polynomials, $\widetilde{P}_{i}(x)$, which are orthonormal to the $P_{i}(x)$; i.e., they satisfy

$$
\int_{-1}^{1} \widetilde{P}_{i}(x, s) P_{j}(x, s) d x=\delta_{i j}
$$

These polynomials are

$$
\begin{aligned}
& \widetilde{P}_{1}(x, s)=\frac{\eta}{2}\left(3 \beta+15 x-15 \beta x^{2}-35 x^{3}\right), \\
& \widetilde{P}_{2}(x, s)=\frac{35}{8}\left(-3 x+5 x^{3}\right), \\
& \widetilde{P}_{3}(x, s)=\frac{5}{8}\left(3+21 z x-9 x^{2}-35 z x^{3}\right), \\
& \widetilde{P}_{4}(x, s)=\frac{\eta}{2}\left(-3-15 z x+15 x^{2}+35 z x^{3}\right) .
\end{aligned}
$$

Notice that $\widetilde{P}_{2}(x)$ is proportional to the third Legendre polynomial.

Thus, the $\sigma_{i}$ are given by

$$
\sigma_{i}=\left[\frac{64 \pi s}{g^{4} \beta}\right] \int_{-1}^{1} d x(z-x)\left(\frac{d \sigma^{a n}}{d x}\right) \widetilde{P}_{i}(x, s),
$$

where the trivial $d \phi$ integration has been carried out.

If we assume that the experimental value $d \sigma^{\exp t} / d \Omega$ for the differential cross section of the above process has been measured, and that all new physics is parametrized by nonstandard trilinear vector couplings, then we have that

$$
\frac{d \sigma_{\text {expt }}^{\text {an }}}{d \Omega}=\frac{d \sigma_{\text {expt }}}{d \Omega}-\frac{d \sigma^{0}}{d \Omega} .
$$

Therefore, the experimental values for $\sigma_{i}$ are given by

$$
\sigma_{i}^{\text {expt }}=\left[\frac{64 \pi s}{g^{4} \beta}\right] \int_{-1}^{1} d x(z-x)\left(\frac{d \sigma^{\text {expt }}}{d x}-\frac{d \sigma^{0}}{d x}\right) \widetilde{P}_{i}(x, s) .
$$

The fact that one can extract, at least in principle, experimental information for the quantities $\sigma_{i}$ motivates the study of the system of equations given in Eq. (3.18). Of course, since Eq. (3.18) constitutes a system of four equations for six unknown quantities, we do not expect to determine all deviation form factors $f_{i}^{V}$ individually. We can easily do so however for two of them; indeed the first and fourth equations in Eqs. (3.18) constitute a separate system of two equations with two unknowns, $f_{3}^{\gamma}$ and $f_{3}^{Z}$, which can be solved exactly:

$$
\begin{aligned}
& f_{3}^{\gamma}=\gamma_{1} \sigma_{1}+\gamma_{4} \sigma_{4}, \\
& f_{3}^{Z}=z_{1} \sigma_{1}+z_{4} \sigma_{4},
\end{aligned}
$$

where

$$
\begin{aligned}
& \gamma_{1}=-\frac{a+v}{2 s_{w}^{4}} \frac{1}{\left[y\left(1-4 c_{w}^{2}\right)+4\right]}, \\
& \gamma_{4}=-\frac{1}{s_{w}^{4}} \frac{\beta\left[y r\left(4 c_{w}^{2}-1\right)+4 s_{w}^{2} v\right]}{\left[y\left(1-4 c_{w}^{2}\right)+4\right]}, \\
& z_{1}=\frac{1}{2 s_{w}^{2} c_{w}^{2}} \frac{1}{y\left[y\left(1-4 c_{w}^{2}\right)+4\right]}, \\
& z_{4}=\frac{1}{s_{w}^{2} c_{w}^{2}} \frac{\beta\left[y v\left(4 c_{w}^{2}-1\right)+4 s_{w}^{2}\right]}{y\left[y\left(1-4 c_{w}^{2}\right)+4\right]} .
\end{aligned}
$$

Thus, the measurement of the two observables $\sigma_{1}$ and $\sigma_{4}$ directly determines the deviations from their SM values, of the magnetic dipole form factors $G_{M}^{\gamma}$ and $G_{M}^{Z}$ of the $W$ due to the photon and the $Z$ respectively: 


$$
\begin{aligned}
& G_{M}^{\gamma}(s)=\frac{e}{2 M_{W}}\left[2+f_{3}^{\gamma}\right]=\frac{e}{2 M_{W}}\left[2+\gamma_{1} \sigma_{1}+\gamma_{4} \sigma_{4}\right], \\
& G_{M}^{Z}(s)=\frac{e}{2 M_{W}} \frac{c_{w}}{s_{w}}\left[2+f_{3}^{Z}\right]=\frac{e}{2 M_{W}} \frac{c_{w}}{s_{w}}\left[2+z_{1} \sigma_{1}+z_{4} \sigma_{4}\right] .
\end{aligned}
$$

We are not aware of the existence in the literature of similar simple expressions relating directly the anomalous magnetic moments to the unpolarized differential cross section with two on-shell $W$ bosons.

The remaining two equations provide two constraints between four of the six non-standard form factors. In particular

$$
\begin{aligned}
a_{1} \sigma_{1}+\sigma_{2}+a_{4} \sigma_{4} & =A_{3} f_{1}^{\gamma}+A_{4} f_{1}^{Z}+A_{7} f_{2}^{\gamma}+A_{8} f_{2}^{Z}, \\
\sigma_{3} & =A_{9} f_{1}^{\gamma}+A_{10} f_{1}^{Z}-\eta A_{9} f_{2}^{\gamma}-\eta A_{10} f_{2}^{Z},
\end{aligned}
$$

where

$$
\begin{aligned}
& a_{1}=-\frac{\beta^{2} \eta}{2} \frac{y\left(1-2 c_{w}^{2}\right)+2}{y\left(1-4 c_{w}^{2}\right)+4}, \\
& a_{4}=-\frac{2 a \beta^{3} \eta y}{y\left(1-4 c_{w}^{2}\right)+4},
\end{aligned}
$$

and we have used that $a-v=s_{w}^{2}$. The above equations can be cast in the equivalent form of two sum rules [11]

$$
\begin{gathered}
\int_{-1}^{1} d x(z-x)\left(\frac{d \sigma^{\text {expt }}}{d x}-\frac{d \sigma^{0}}{d x}\right) H(x, s) \\
=A_{3} f_{1}^{\gamma}+A_{4} f_{1}^{Z}+A_{7} f_{2}^{\gamma}+A_{8} f_{2}^{Z}, \\
\int_{-1}^{1} d x(z-x)\left(\frac{d \sigma^{\text {expt }}}{d x}-\frac{d \sigma^{0}}{d x}\right) \widetilde{P}_{3}(x, s) \\
=A_{9} f_{1}^{\gamma}+A_{10} f_{1}^{Z}-\eta A_{9} f_{2}^{\gamma}-\eta A_{10} f_{2}^{Z},
\end{gathered}
$$

where

$$
H(x, s)=a_{1} \widetilde{P}_{1}(x, s)+\widetilde{P}_{2}(x, s)+a_{4} \widetilde{P}_{4}(x, s) .
$$

The analysis and the results presented thus far are model independent since no assumptions have been made about the dynamical mechanism which gives rise to the anomalous couplings. It would be interesting to examine how the above results could be used for testing the validity of specific models which predict the generation of such couplings. We will discuss some of these issues in the next section, in the context of a composite model for the $W$.

\section{PREDICTIONS OF A COMPOSITE MODEL}

In this section we examine the predictions at the level of experimental cross sections of a model of compositeness proposed by Brodsky and Hiller [12]. In this model the $W$ is
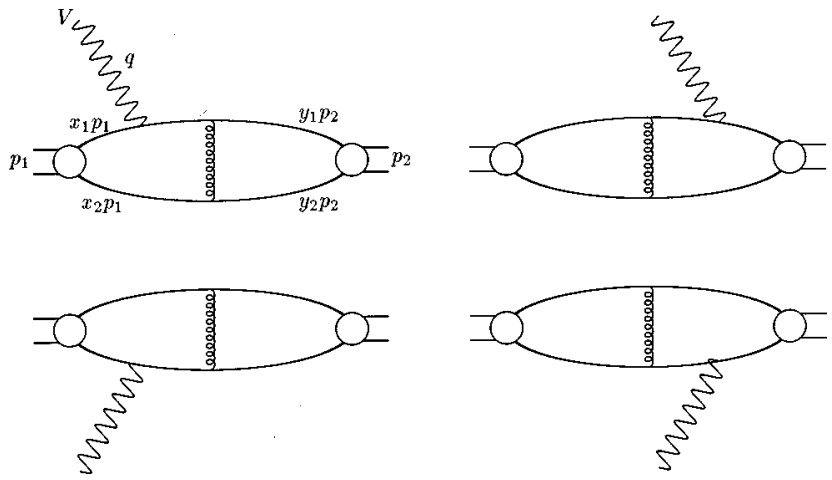

FIG. 2. The composite model in the one-boson exchange approximation.

considered a bound state of two, in general different, fermions. The two fermions are held together by the exchange of a gauge boson of mass $\lambda$. The results presented have been calculated in the one-boson exchange approximation (Fig. 2 ); as explained in Ref. [12] the model becomes gauge invariant in the collinear approximation.

The matrix element $\left(G_{h, h^{\prime}}^{V}\right)_{\mu}=\left\langle p_{2}, h^{\prime}\left|J_{V}^{\mu}\right| p_{1}, h\right\rangle$ of the current $J_{V}^{\mu}$ between the momentum and helicity eigenstates $\left|p_{1}, h\right\rangle$ and $\left|p_{2}, h^{\prime}\right\rangle$ is written in terms of three-form factors ${ }^{1}$ as follows:

$$
\begin{aligned}
\left(G_{h, h^{\prime}}^{V}\right)_{\mu}= & -G_{1}^{V}\left(q^{2}\right)\left(\epsilon^{* *} \cdot \boldsymbol{\epsilon}\right)\left(p_{2}-p_{1}\right)_{\mu} \\
& -G_{2}^{V}\left(q^{2}\right)\left[\left(\epsilon^{\prime *} \cdot q\right) \epsilon_{\mu}-(\epsilon \cdot q) \epsilon_{\mu}^{*}\right] \\
& +G_{3}^{V}\left(q^{2}\right) / 2 M_{W}^{2}\left(\epsilon^{\prime *} \cdot q\right)(\epsilon \cdot q)\left(p_{2}-p_{1}\right)_{\mu}
\end{aligned}
$$

where $\epsilon \equiv \epsilon_{h}$ and $\epsilon^{\prime} \equiv \epsilon_{h^{\prime}}$ are the initial and final polarization vectors.

The kinematical form factors $G_{1}^{V}, G_{2}^{V}$, and $G_{3}^{V}$ are related to the photonic $(V=\gamma)$ or weak $(V=Z)$ charge $G_{C}^{V}$, magnetic dipole $G_{M}^{V}$, and electric quadruple $G_{Q}^{V}$ form factors of the $W$ through the relations

$$
\begin{aligned}
& G_{C}^{V}=G_{1}^{V}+\frac{2 \eta}{3} G_{Q}^{V}, \\
& G_{M}^{V}=G_{2}^{V}, \\
& G_{Q}^{V}=G_{1}^{V}-G_{2}^{V}+(1+\eta) G_{3}^{V} .
\end{aligned}
$$

As was shown in Ref. [12], this model predicts the following ratios for photon form factors:

$$
G_{C}^{\gamma}: G_{M}^{\gamma}: G_{Q}^{\gamma}=\left(1-\frac{2}{3} \eta\right): 2:-1
$$

\footnotetext{
${ }^{1}$ We use the same notation as in Ref. [12], except for the labelling of the four-momenta, where we have set $p^{\prime} \rightarrow p_{2}$ and $p \rightarrow-p_{1}$.
} 
which, in the notation of Eq. (1), translates into

$$
f_{2}^{\gamma}=0, \quad f_{1}^{\gamma}=f_{3}^{\gamma}
$$

Notice that the above ratios, which have been derived using the non-trivial dynamics of this composite model, coincide with the corresponding ratios satisfied by the tree-level SM values of $G_{C}, G_{M}$, and $G_{Q}$.

However, no analogous ratios for the form factors $G_{C}^{Z}$, $G_{M}^{Z}$, and $G_{Q}^{Z}$ were derived in Ref. [12], because their analysis had focused on the photon form factors only. In what follows we will address this issue in some detail. In particular we will study how the anomalous gauge boson couplings predicted by this model are affected by the coupling of the neutral gauge bosons $V(V=\gamma, Z)$ to the fermions, which make up the composite $W$.

The most general coupling of the $i$ th fermion $(i=1,2)$ allowed by Lorentz invariance is given by

$$
\Gamma_{\mu}^{V, i}=\gamma_{\mu} F_{1}^{V, i}+\sigma_{\mu \nu} q^{\nu} F_{2}^{V, i}+\gamma_{\mu} \gamma_{5} F_{3}^{V, i}+\sigma_{\mu \nu} q^{\nu} \gamma_{5} F_{4}^{V, i},
$$

where $\sigma_{\mu \nu}=(i / 2)\left[\gamma_{\mu}, \gamma_{\nu}\right]$. Note that $F_{1}^{V, i}$ and $F_{3}^{V, i}$ are dimensionless quantities, whereas $F_{2}^{V, i}$ and $F_{4}^{V, i}$ have dimensions of inverse mass. To determine the effect of this general fermion-boson coupling on the anomalous trilinear gauge boson couplings we must repeat the calculation presented in Ref. [12], keeping the general form for $\Gamma_{\mu}^{V, i}$ given on the left hand side of Eq. (4.5), instead of only the term proportional to $\gamma_{\mu}$.

From Ref. [12] we know that

$$
\left(G_{h, h^{\prime}}^{V}\right)_{\mu} \sim \frac{1}{s} \frac{1}{\left(s-4 \lambda^{2}\right) i=1,2} \sum_{h h^{\prime}}\left[\left(A_{\mu}^{V, i}\right)_{\mu}+\left(B_{h h^{\prime}}^{V, i}\right)_{\mu}\right],
$$

with

$$
\begin{aligned}
& \left(A_{h h^{\prime}}^{V, i}\right)_{\mu}=\operatorname{Tr}\left\{\gamma_{\nu} \bar{\chi}_{J h^{\prime}} \gamma^{\nu}\left[p_{2}-\frac{1}{2}\left(p_{1}-M\right)\right] \Gamma_{\mu}^{V, i} \chi_{J h}\right\}, \\
& \left(B_{h h^{\prime}}^{V, i}\right)_{\mu}=-\operatorname{Tr}\left\{\bar{\chi}_{J h^{\prime}} \Gamma_{\mu}^{V, i}\left[p_{1}+\frac{1}{2}\left(p_{2}-M\right)\right] \gamma^{\nu} \chi_{J h} \gamma_{\nu}\right\},
\end{aligned}
$$

where $M$ is the mass of the composite $W$, and the spin wave functions are given by

$$
\chi_{1 h}=\frac{-1}{\sqrt{2}} k_{h}(\not p-M), \quad \chi_{00}=\frac{1}{\sqrt{2}} \gamma_{5}(\not p-M) .
$$

If we define the quantity

$$
\widetilde{F}_{j}^{V}=\sum_{i} F_{j}^{V, i}, \quad j=1,2,3,4
$$

we find in the limit where the mass $M$ of the composite $W$ and the masses $m_{1}$ and $m_{2}$ of the constituent fermions satisfy $M=m_{1}+m_{2}\left(x_{1}=x_{2}=y_{1}=y_{2}=1 / 2\right.$ in the notation of [12]):

$$
\begin{aligned}
& f_{1}^{V}=\widetilde{F}_{1}^{V}-\frac{2 s}{M} \widetilde{F}_{2}^{V}-1, \\
& f_{2}^{V}=-4 M \widetilde{F}_{2}^{V}, \\
& f_{3}^{V}=\widetilde{F}_{1}^{V}-\left(\frac{s+4 M^{2}}{2 M}\right) \widetilde{F}_{2}^{V}-1, \\
& f_{4}^{V}=0, \\
& f_{5}^{V}=-i \widetilde{F}_{3}^{V}+i\left(\frac{s}{2 M}\right) \widetilde{F}_{4}^{V}, \\
& f_{6}^{V}=4 M \widetilde{F}_{4}^{V}, \\
& f_{7}^{V}=2 M \widetilde{F}_{4}^{V} .
\end{aligned}
$$

In deriving these results we have made use of the identities listed in Eq. (A3) and Eq. (A4) in the Appendix of Ref. [6]. We see that the presence of $\widetilde{F}_{2}^{V}$ distorts the compositeness condition, whereas $\widetilde{F}_{3}^{V}$ and $\widetilde{F}_{4}^{V}$ do not enter in the definition of $f_{1}^{V}, f_{2}^{V}$ and $f_{3}^{V}$.

Having established the above results we will now pursue two different possibilities: (i) We will assume that the compositeness condition holds for the photonic form factors only, i.e., $\widetilde{F}_{2}^{\gamma}=0$ but $\widetilde{F}_{2}^{Z} \neq 0$. (ii) We will assume that the compositeness condition holds for both the photonic and $Z$ form factors, i.e., $\widetilde{F}_{2}^{\gamma}=\widetilde{F}_{2}^{Z}=0$.

In the first case, using Eq. (4.4), which we assume to be true, we obtain from Eq. (3.27) and Eq. (3.30) the following system of two equations for the two remaining unknown form factors $f_{1}^{Z}, f_{2}^{Z}$ :

$$
\begin{gathered}
\left(a_{1}-A_{3} \gamma_{1}\right) \sigma_{1}+\sigma_{2}+\left(a_{4}-A_{3} \gamma_{4}\right) \sigma_{4} \\
=A_{4} f_{1}^{Z}+A_{8} f_{2}^{Z} \\
-A_{9} \gamma_{1} \sigma_{1}+\sigma_{2}-A_{9} \gamma_{4} \sigma_{4} \\
=A_{10} f_{1}^{Z}-\eta A_{10} f_{2}^{Z} .
\end{gathered}
$$

This yields the solutions

$$
\begin{aligned}
f_{1}^{Z=} & \sigma_{1}\left(\gamma_{1} b_{1}-\eta a_{1} A_{10}\right) / D_{1}-\sigma_{2} \eta A_{10} / D_{1}-\sigma_{3} A_{8} / D_{1} \\
& +\sigma_{4}\left(\gamma_{4} b_{1}-\eta a_{4} A_{10}\right) / D_{1} \\
f_{2}^{Z}= & \sigma_{1}\left(\gamma_{1} b_{2}-a_{1} A_{10}\right) / D_{1}-\sigma_{2} A_{10} / D_{1}+\sigma_{3} A_{4} / D_{1} \\
& +\sigma_{4}\left(\gamma_{4} b_{2}-a_{4} A_{10}\right) / D_{1}
\end{aligned}
$$

with

$$
\begin{aligned}
& b_{1}=\eta A_{10} A_{3}+A_{8} A_{9}, \\
& b_{2}=A_{10} A_{3}-A_{4} A_{9},
\end{aligned}
$$




$$
D_{1}=(a+v) \beta^{3} \eta y^{2} c_{w}^{4}\left[y r\left(2 c_{w}^{2}-1\right)+2 v s_{w}^{2}\right] .
$$

Thus, assuming that the couplings of $W$ to the photon obey their tree-level SM relations one can extract all the remaining of the $C$ and $P$ conserving anomalous form factors directly from the differential cross section.

In the second possibility of interest, using the fact that, because of the compositeness conditions $f_{2}^{V}=0$, Eq. (3.30) reduces to

$$
\begin{aligned}
a_{1} \sigma_{1}+\sigma_{2}+a_{4} \sigma_{4} & =A_{3} f_{1}^{\gamma}+A_{4} f_{1}^{Z}, \\
\sigma_{3} & =A_{9} f_{1}^{\gamma}+A_{10} f_{1}^{Z} .
\end{aligned}
$$

Then, since $f_{1}^{V}=f_{3}^{V}$, we can substitute into Eq. (4.14) the solutions for $f_{3}^{V}$ from Eq. (3.27) to arrive at

$$
\begin{aligned}
\sigma_{2}+c_{1} \sigma_{1}+c_{2} \sigma_{4} & =0, \\
\sigma_{3}+c_{3} \sigma_{4} & =0,
\end{aligned}
$$

where

$$
\begin{aligned}
& c_{1}=\frac{1}{8} \beta^{2} \frac{2(3-2 \eta)\left(1-y c_{w}^{2}\right)+(1-2 \eta) y}{4\left(1-y c_{w}^{2}\right)+y}, \\
& c_{2}=-\frac{a}{2} \beta^{3} y \frac{(1+6 \eta)}{4\left(1-y c_{w}^{2}\right)+y}, \\
& c_{3}=\frac{\beta^{2}}{4} .
\end{aligned}
$$

Using Eq. (4.16) and assuming Eq. (3.25) we obtain the following two predictions:

$$
\int_{-1}^{1} d x(z-x)\left(\frac{d \sigma^{e x p t}}{d x}-\frac{d \sigma^{0}}{d x}\right) H_{i}(x, s)=0, \quad i=1,2,
$$

where $H_{1}$ and $H_{2}$ are polynomials in $x$ of maximum degree 3 , given by

$$
\begin{aligned}
& H_{1}=\widetilde{P}_{2}+c_{1} \widetilde{P}_{1}+c_{2} \widetilde{P}_{4}, \\
& H_{2}=\widetilde{P}_{3}+c_{3} \widetilde{P}_{4} .
\end{aligned}
$$

\section{PREDICTIONS OF AN EFFECTIVE LAGRANGIAN APPROACH}

We now turn our attention to an effective Lagrangian approach to anomalous gauge couplings based on $S U(2)_{L}$ $\times U(1)_{Y}$ gauge invariance. In such an approach the deviations of the gauge couplings arise from gauge invariant but non-standard interaction terms of dimension $d>4$, between gauge bosons and the Higgs field [13-17]. Such terms are assumed to originate from an as yet unknown underlying theory at a new-physics mass scale $\Lambda$. Thus, the corresponding strengths of these interactions will be suppressed by factors $(\Lambda)^{4-d}$.

In order for all form factors of the vertex of Eq. (2.6) to be generated one must consider a host of operators of dimension $d$ up to 12; in such a scenario no constraints can be obtained among the various form factors. If on the other hand the new-physics mass scale $\Lambda$ is assumed to be large, e.g., $\Lambda \geqslant 1 \mathrm{TeV}$, a low energy approximation where only operators of dimension 6 are retained will lead to relations among the various otherwise unrelated form factors of the vertex. Such relations were first derived by imposing global $S U(2)$ symmetry on the phenomenological Lagrangian that generates the vertex of Eq. (2.6) $[13,30]$. In fact, the precise nature of these relations depends on whether a linear or nonlinear realization of the symmetry is adopted. For a relatively light Higgs the new physics is described by a linear realization of the symmetry, while for a sufficiently heavy one a non-linear realization is required.

We consider first a linear realization of the Higgs sector. The basic ingredients of an $S U(2)_{L} \times U(1)_{Y}$ gauge invariant Lagrangian are the Higgs field $\Phi$, its covariant derivative $D_{\mu} \Phi$, and the non-Abelian field strengths $B_{\mu \nu}$ and $\mathbf{W}_{\mu \nu}$ of the $U(1)_{Y}$ and $S U(2)_{L}$ gauge fields, respectively. If one imposes the additional requirement of separate $C$ and $P$ invariance, there are three operators of dimension 6 that can induce trilinear gauge couplings. They are described by the effective Lagrangian

$$
\begin{aligned}
\mathcal{L}_{\text {eff }}^{d=6}= & i g \frac{\alpha_{B \phi}}{M_{w}^{2}}\left(D_{\mu}\right)^{\dagger} B^{\mu \nu}\left(D_{\nu}\right)+i g \frac{\alpha_{W \phi}}{M_{w}^{2}}\left(D_{\mu}\right)^{\dagger} \tau \cdot \mathbf{W}^{\mu \nu}\left(D_{\nu}\right) \\
& +g \frac{\alpha_{W}}{6 M_{w}^{2}} \mathbf{W}_{\nu}^{\mu} \cdot\left(\mathbf{W}_{\rho}^{\nu} \times \mathbf{W}_{\mu}^{\rho}\right),
\end{aligned}
$$

where $g$ and $g^{\prime}$ are the $S U(2)_{L}$ and $U(1)_{Y}$ gauge couplings, respectively.

The part of the above Lagrangian describing the selfinteractions of the gauge bosons is obtained by replacing the Higgs field with its vacuum expectation value, $\Phi^{T}$ $\rightarrow(0, u / \sqrt{2})$. Explicitly

$$
\begin{aligned}
\mathcal{L}_{\text {eff }}^{W W V}= & i g_{V}\left\{g_{1}^{V}\left(W_{\mu \nu}^{+} W^{-\mu}-W^{+\mu} W_{\mu \nu}^{-}\right) V^{\nu}+\kappa_{V} W_{\mu}^{+} W_{\nu}^{-} V^{\mu \nu}\right. \\
& \left.+\frac{\lambda_{V}}{M_{w}^{2}} W_{\mu}^{+\nu} W_{\nu}^{-\rho} V_{\rho}^{\mu}\right\}
\end{aligned}
$$

In the SM the couplings $g_{1}^{V}, \kappa_{V}$ and $\lambda_{V}$ have the values $g_{1}^{V}=\kappa_{V}=1, \lambda_{V}=0$, and are directly related to the charge, magnetic dipole, and electric quadruple moments of the $W$. Here $g_{1}^{\gamma}$ is fixed to 1 by electromagnetic gauge invariance, while the rest of the couplings are parametrized by the available free parameters of the effective Lagrangian $\alpha_{W \phi}, \alpha_{B \phi}$, and $\alpha_{W}$ according to

$$
\Delta g_{1}^{Z}=\alpha_{W \phi} / c_{w}^{2},
$$




$$
\begin{aligned}
\Delta \kappa_{\gamma} & =\alpha_{W \phi}+\alpha_{B \phi}, \\
\Delta \kappa_{Z} & =\alpha_{W \phi}-\frac{s_{w}^{2}}{c_{w}^{2}} \alpha_{B \phi}, \\
\lambda_{\gamma} & =\alpha_{W}, \\
\lambda_{Z} & =\alpha_{W} .
\end{aligned}
$$

Since no operators containing derivatives of the form $\square^{n} V^{\mu}$ (or $\square^{n} W^{\alpha}$ for off-shell $W^{\prime}$ s) are present in Eq. (5.2), the resulting form factors will be strictly constants, independent of $s$. Nevertheless they must scale as $M_{W}^{2} / \Lambda^{2}$. The additional requirement of tree level unitarity imposes bounds on the products of the $\alpha_{i}$ with the scale $\Lambda[28,31]$.

The relations of Eq. (5.3) constitute the constraints predicted by this model for the anomalous gauge couplings. They may also be found in the literature in the following form:

$$
\begin{gathered}
\Delta \kappa_{Z}=\Delta g_{1}^{Z}-\frac{s_{w}^{2}}{c_{w}^{2}} \Delta \kappa_{\gamma}, \\
\lambda_{\gamma}=\lambda_{Z} .
\end{gathered}
$$

In order to translate them into relations among the $f_{i}$ form factors, we must use that [6]

$$
\begin{gathered}
f_{1}^{V}=\Delta g_{1}^{V}+\frac{s}{2 m_{w}^{2}} \lambda_{V}, \\
f_{2}^{V}=2 \lambda_{V}, \\
2 f_{3}^{V}=\Delta g_{1}^{V}+\Delta \kappa_{V}+\lambda_{V} .
\end{gathered}
$$

Then Eq. (5.3) gives rise to the following constraints on $f_{2}^{\gamma}, f_{3}^{\gamma}$, and $f_{1}^{Z}$ :

$$
\begin{gathered}
f_{1}^{\gamma}=\eta f_{2}^{\gamma}, \\
f_{2}^{Z}=f_{2}^{\gamma}, \\
s_{w}^{2} f_{3}^{\gamma}+c_{w}^{2} f_{3}^{Z}=c_{w}^{2} f_{1}^{Z}+\rho f_{2}^{\gamma},
\end{gathered}
$$

where $\rho=2 \eta c_{w}^{2}-\frac{1}{2}$.

We now return to the system of Eq. (3.30) and determine what the relations given above imply for the $\sigma_{i}$ observables. Feeding the first two relations of Eq. (5.6) into the system of equations (3.30) the latter can now be solved for the two unknown quantities $f_{1}^{Z}$ and $f_{2}^{\gamma}$ :

$$
\begin{gathered}
a_{1} \sigma_{1}+\sigma_{2}+a_{4} \sigma_{4}=\left(\eta A_{3}+A_{7}+A_{8}\right) f_{2}^{\gamma}+A_{4} f_{1}^{Z}, \\
\sigma_{3}=\eta\left(A_{9}-A_{10}\right) f_{2}^{\gamma}+A_{10} f_{1}^{Z} .
\end{gathered}
$$

Defining

$$
B_{1}=\eta A_{3}+A_{7}+A_{8},
$$

$$
\begin{aligned}
B_{2} & =\eta\left(A_{9}-A_{10}\right), \\
\Delta & =B_{1} A_{10}-B_{2} A_{4},
\end{aligned}
$$

we obtain the solution

$$
\begin{aligned}
& f_{2}^{\gamma}=-\left[a_{1} A_{10} \sigma_{1}+A_{10} \sigma_{2}-A_{4} \sigma_{3}+a_{4} A_{10} \sigma_{4}\right] \Delta^{-1}, \\
& f_{1}^{Z}=-\left[a_{1} B_{2} \sigma_{1}+B_{2} \sigma_{2}-B_{1} \sigma_{3}+a_{4} B_{2} \sigma_{4}\right] \Delta^{-1} .
\end{aligned}
$$

Finally, substituting the above solutions and the solutions for $f_{3}^{\gamma}, f_{3}^{Z}$ from Eq. (3.27) into the constraint of the third relation of Eq. (5.6) the latter assumes the following form in terms of the $\sigma_{i}$ observables:

$$
h_{1} \sigma_{1}+h_{2} \sigma_{2}+h_{3} \sigma_{3}+h_{4} \sigma_{4}=0 \text {, }
$$

where

$$
\begin{aligned}
& h_{1}=\Delta\left(s_{w}^{2} \gamma_{1}+c^{2} z_{1}\right)+a_{1}\left(c_{w}^{2} B_{2}-\rho A_{10}\right), \\
& h_{2}=c_{w}^{2} B_{2}-\rho A_{10}, \\
& h_{3}=-c_{w}^{2} B_{1}+\rho A_{4}, \\
& h_{4}=\Delta\left(s_{w}^{2} \gamma_{4}+c^{2} z_{4}\right)+a_{4}\left(c_{w}^{2} B_{2}-\rho A_{10}\right) .
\end{aligned}
$$

Equation (5.10) constitutes the prediction of this approach; it can be cast in the alternative form

$$
\int_{-1}^{1} d x(z-x)\left(\frac{d \sigma^{e x p t}}{d x}-\frac{d \sigma^{0}}{d x}\right) H_{3}(x, s)=0
$$

with

$$
H_{3}(x, s)=h_{1} \widetilde{P}_{1}+h_{2} \widetilde{P}_{2}+h_{3} \widetilde{P}_{3}+h_{4} \widetilde{P}_{4} .
$$

A particular case of the linear realization of the symmetry is the so called Hagiwara-Ishihara-Szalapski-Zeppenfeld (HISZ) scenario, proposed in Ref. [15], where the third operator (usually denoted $\mathcal{O}_{W W W}$ ) in Eq. (5.1) is missing. Equivalently, one sets $a_{W}=0$ or $\lambda_{V}=0$ in Eq. (5.2). Then the relations imposed on the anomalous couplings become

$$
\begin{array}{r}
f_{1}^{\gamma}=f_{2}^{\gamma}=f_{2}^{Z}=0, \\
s_{w}^{2} f_{3}^{\gamma}+c_{w}^{2} f_{3}^{Z}=c_{w}^{2} f_{1}^{Z},
\end{array}
$$

and Eq. (3.30) transforms to the over-constrained system

$$
\begin{aligned}
a_{1} \sigma_{1}+\sigma_{2}+a_{4} \sigma_{4} & =A_{4} f_{1}^{Z}, \\
\sigma_{3} & =A_{10} f_{1}^{Z} .
\end{aligned}
$$

This provides the solution

$$
f_{1}^{Z}=\frac{\sigma_{3}}{A_{10}}
$$


and the additional constraint

$$
a_{1} A_{10} \sigma_{1}+A_{10} \sigma_{2}-A_{4} \sigma_{3}+a_{4} A_{10} \sigma_{4}=0 .
$$

Evidently in this case where the electric charge form factor has been strictly set to 1 while, at the same time $f_{2}^{Z}=0, \sigma_{3}$ measures directly the weak charge of the $W$.

The initial constraint of Eq. (5.15) in terms of the $\sigma_{i}$ reads

$$
\left(s_{w}^{2} \gamma_{1}+c_{w}^{2} z_{1}\right) \sigma_{1}-\frac{c_{w}^{2}}{A_{10}} \sigma_{3}+\left(s_{w}^{2} \gamma_{4}+c_{w}^{2} z_{4}\right) \sigma_{4}=0 .
$$

Both constraints can be cast in the equivalent form

$$
\int_{-1}^{1} d x(z-x)\left(\frac{d \sigma^{e x p t}}{d x}-\frac{d \sigma^{0}}{d x}\right) H_{i}(x, s)=0, \quad i=4,5
$$

with

$$
\begin{aligned}
& H_{4}(x, s)=a_{1} A_{10} \widetilde{P}_{1}+A_{10} \widetilde{P} 2-A_{4} \widetilde{P}_{3}+a_{4} A_{10} \widetilde{P}_{4}, \\
& H_{5}(x, s)=\left(s_{w}^{2} \gamma_{1}+c_{w}^{2} z_{1}\right) \widetilde{P}_{1}-\frac{c_{w}^{2}}{A_{10}} \widetilde{P}_{3}+\left(s_{w}^{2} \gamma_{4}+c_{w}^{2} z_{4}\right) \widetilde{P}_{4} .
\end{aligned}
$$

In the very heavy Higgs boson case (or equivalently if the Higgs boson is absent), where the symmetry is realized nonlinearly, the Higgs doublet is replaced by a unitary matrix $U \equiv \exp (i \omega \cdot \tau / v)$, where the $\omega_{i}$ are the would-be Goldstone bosons, and the appropriate matrix form of the covariant derivative is implied. It is easy to see that the sum rule obtained in this case assumes again the simple form of Eq. (5.20). Indeed, naive dimensional analysis [32] suggests that the $\lambda_{V}$ couplings are suppressed by additional powers of the new physics scale $\left(M_{W}^{4} / \Lambda^{4}\right)$ and are expected to be negligible with respect to $\Delta g_{1}^{V}$ and $\Delta \kappa_{V}$. Thus they are set to zero and there remain again three free parameters $\Delta g_{1}^{Z}, \Delta \kappa_{\gamma}$ and $\Delta \kappa_{Z}$. Accordingly, for the $f_{i}$ we obtain the relation of Eq. (5.14), but not the second relation of Eq. (5.15), and the constraint becomes simply

$$
\int_{-1}^{1} d x(z-x)\left(\frac{d \sigma^{e x p t}}{d x}-\frac{d \sigma^{0}}{d x}\right) H_{4}(x, s)=0 .
$$

\section{STATISTICAL PROPERTIES OF THE $\sigma_{i}$ OBSERVABLES}

In this section we present an elementary discussion of the statistical properties of the observables proposed. In particular we study the covariance matrix and correlation functions for the $\sigma_{i}$ observables and the error propagation to the form factors $f_{i}$. This discussion is meant to provide a rough idea about the feasibility of the proposed measurements, especially in connection with the ongoing LEP2 experiments. In general, the most important sources of error come from the resolution in the reconstruction of the $W$ and from the back- grounds in the selected sample; a more detailed error analysis based on real experimental data is underway [33].

The following analysis is based on the maximum likelihood method [34]. A probability density $p(x)$ may be constructed by means of the differential cross section $d \sigma / d x$ and the total cross section $\sigma$, where

$$
\sigma=\int_{-1}^{1} d x\left(\frac{d \sigma}{d x}\right)
$$

by setting

$$
p(x)=\frac{1}{\sigma}\left(\frac{d \sigma}{d x}\right)
$$

Clearly,

$$
\int_{-1}^{1} d x p(x)=1
$$

We may then interpret the observables $\sigma_{i}$ as the expectation values corresponding to a set of local observable $O_{i}(x)$ given by

$$
O_{i}(x)=\left[\frac{64 \pi s \sigma}{g^{4} \beta}\right](z-x) \widetilde{P}_{i}(x, s),
$$

i.e.,

$$
\sigma_{i}=\int_{-1}^{1} d x p(x) O_{i}(x)
$$

If we adopt this interpretation, then the correlation matrix $V_{i j}$ of the $\sigma_{i}$ observables is given by

$$
\begin{aligned}
V_{i j}= & \int_{-1}^{1} d x p(x)\left[O_{i}(x)-\sigma_{i}\right]\left[O_{j}(x)-\sigma_{j}\right] \\
= & \int_{-1}^{1} d x p(x) O_{i}(x) O_{j}(x)-\left[\int_{-1}^{1} d x p(x) O_{i}(x)\right] \\
& \times\left[\int_{-1}^{1} d x p(x) O_{j}(x)\right]
\end{aligned}
$$

and the correlation function by

$$
\rho_{i j}=\frac{V_{i j}}{\left(V_{i i} V_{j j}\right)^{1 / 2}} .
$$

Some typical values of the above quantities for different values of the center of mass energy $\sqrt{s}$ are shown in Table II. The values for $\sigma$ and $d \sigma / d x$ we use when computing $V_{i j}$ and $\rho_{i j}$ are those of the SM in the absence of the anomalous coupling, i.e., $d \sigma^{0} / d x$ and $\sigma^{0}$ given in Ref. [29]; this assumes implicitly that the experimental data are well described by the SM [35]. We have used $M_{W}=80 \mathrm{GeV}$ and $M_{Z}=91 \mathrm{GeV}$. 
TABLE II. The covariance matrix $V_{i j}$ and the correlation coefficients $\rho_{i j}$ as a function of the center-of-mass energy $\sqrt{s}$ (in GeV).

\begin{tabular}{lcccccccccccc}
\hline \hline$\sqrt{s}$ & 165 & 170 & 175 & 180 & 185 & 190 & 195 & 200 & 250 & 300 & 400 & 500 \\
\hline$V_{11}$ & 645 & 329 & 227 & 178 & 151 & 134 & 123 & 116 & 127 & 203 & 581 & 1482 \\
$V_{22}$ & 695 & 346 & 230 & 173 & 140 & 117 & 101 & 90 & 47 & 37 & 32 & 31 \\
$V_{33}$ & 3181 & 898 & 448 & 282 & 201 & 155 & 126 & 107 & 48 & 38 & 33 & 33 \\
$V_{44}$ & 2974 & 867 & 452 & 299 & 226 & 185 & 161 & 146 & 139 & 218 & 611 & 1535 \\
$V_{12}$ & -561 & -283 & -192 & -148 & -122 & -105 & -94 & -86 & -63 & -68 & -98 & -148 \\
$V_{13}$ & 1190 & 451 & 266 & 188 & 147 & 122 & 106 & 96 & 70 & 78 & 122 & 192 \\
$V_{14}$ & -1367 & -522 & -311 & -224 & -178 & -152 & -136 & -126 & -130 & -209 & -594 & -1508 \\
$V_{23}$ & -1446 & -530 & -301 & -205 & -153 & -122 & -102 & -88 & -42 & -33 & -28 & -28 \\
$V_{24}$ & 1175 & 440 & 256 & 179 & 139 & 114 & 99 & 88 & 61 & 65 & 96 & 147 \\
$V_{34}$ & -2612 & -759 & -391 & -255 & -189 & -151 & -128 & -113 & -75 & -82 & -125 & -194 \\
$\rho_{12}$ & -0.837 & -0.838 & -0.840 & -0.841 & -0.842 & -0.842 & -0.841 & -0.841 & -0.815 & -0.782 & -0.731 & -0.700 \\
$\rho_{13}$ & 0.831 & 0.830 & 0.832 & 0.836 & 0.841 & 0.847 & 0.852 & 0.859 & 0.895 & 0.901 & 0.886 & 0.870 \\
$\rho_{14}$ & -0.987 & -0.978 & -0.971 & -0.968 & -0.966 & -0.966 & -0.966 & -0.967 & -0.982 & -0.992 & -0.998 & -0.999 \\
$\rho_{23}$ & -0.972 & -0.952 & -0.937 & -0.924 & -0.915 & -0.908 & -0.902 & -0.898 & -0.880 & -0.878 & -0.880 & -0.883 \\
$\rho_{24}$ & 0.817 & 0.804 & 0.794 & 0.788 & 0.782 & 0.778 & 0.775 & 0.772 & 0.750 & 0.732 & 0.702 & 0.682 \\
$\rho_{34}$ & -0.849 & -0.806 & -0.870 & -0.879 & -0.886 & -0.893 & -0.898 & -0.902 & -0.915 & -0.907 & -0.885 & -0.868 \\
\hline \hline
\end{tabular}

The symmetric $2 \times 2$ correlation matrix $F_{i j}(i, j=Z, \gamma)$ for $f_{3}^{\gamma}$ and $f_{3}^{Z}$ is given in terms of the $2 \times 2$ correlation matrix $\hat{V}_{i j}$ of $\sigma_{1}$ and $\sigma_{4}$ by $[35,36]$

$$
F_{i j}=N^{-1}\left(\Lambda \hat{V} \Lambda^{T}\right)_{i j}
$$

where $N$ is the number of observed events, and $\Lambda_{11}=\gamma_{1}$, $\Lambda_{12}=\gamma_{4}, \Lambda_{21}=z_{1}, \Lambda_{22}=z_{4}\left[\gamma_{1}, \gamma_{4}, z_{1}\right.$, and $z_{4}$ are given in Eq. (3.28)] whereas the entries of the matrix $\hat{V}_{i j}$ are given by $\hat{V}_{11}=V_{11}, \hat{V}_{12}=\hat{V}_{21}=V_{14}$ and $\hat{V}_{22}=V_{44}$. Finally, the superscript $T$ means 'transposed.' Some characteristic values of the $F_{i j}$ and the corresponding errors obtained from it are shown in Table III as a function of the center-of-mass energy $\sqrt{s}$ (in $\mathrm{GeV}$ ).

The following points are worth mentioning:

(a) From Table II we see that the observables $\sigma_{i}$ display a rather high degree of correlation (anti-correlation). We notice also that the (anti-)correlation does not change significantly as a function of $\sqrt{s}$.

(b) The high anti-correlation between $\sigma_{1}$ and $\sigma_{4}$ carries over to $f_{3}^{\gamma}$ and $f_{3}^{Z}$; indeed, as shown in Table III, the two deviation form factors are almost completely anti-correlated.

(c) The (absolute) values of the entries of $V_{i j}$ and $F_{i j}$ vary significantly as functions of $\sqrt{s}$. The minimum value for all
$V_{i j}$ is reached at about $300 \mathrm{GeV}$. At that point the error in $f_{3}^{\gamma}$ and $f_{3}^{Z}$, namely $\epsilon_{\gamma}$ and $\epsilon_{z}$ respectively, are a factor of 2 smaller than the corresponding error in the LEP2 energy range $(165-200 \mathrm{GeV})$. After reaching their minimum value the errors increase monotonically with increasing $\sqrt{s}$. To get a rough estimate of the error for LEP2 energies, for $\sqrt{s}$ $=190 \mathrm{GeV}$ we have $\epsilon_{\gamma}=0.85, \epsilon_{z}=0.6$ for $N=5000$, and $\epsilon_{\gamma}=0.6, \epsilon_{z}=0.4$ for $N=10000$.

(d) From Table I we observe that below $180 \mathrm{GeV}$ the (absolute) values of the coefficients $A_{i}(i=3, \ldots, 10)$ which enter in the right hand side of the equations for $\sigma_{2}$ and $\sigma_{3}$ in Eq. (3.18) are significantly smaller than the coefficients $A_{1}$, $A_{2}$ and $A_{11}, A_{12}$, entering in the equations for $\sigma_{1}$ and $\sigma_{4}$ respectively. Given that the errors in $\sigma_{2}$ and $\sigma_{3}$, i.e., $\left(V_{22}\right)^{1 / 2}$ and $\left(V_{33}\right)^{1 / 2}$, appear to be comparable to those for $\sigma_{1}$ and $\sigma_{4}$, i.e., $\left(V_{11}\right)^{1 / 2}$ and $\left(V_{44}\right)^{1 / 2}$, one would tend to conclude that the efficiency of the two sum rules in Eq. (3.18) may be degraded considerably. This issue needs further investigation; in particular one should study (i) whether the above conclusion persists a more realistic error analysis based on real experimental data and (ii) if so, whether further experimental inputs, e.g., studying the $W$ decay angular distributions, may ameliorate the situation.

(e) By observing Table I we see that the ratio $A_{1} / A_{2}$ has a significant dependence on $\sqrt{s}$; it grows from 0.55 at $\sqrt{s}$

TABLE III. The coefficients $F_{i j}$, the correlation factor $\rho$, and the corresponding errors $\left[\epsilon_{\gamma}=\left(F_{\gamma \gamma}\right)^{1 / 2}, \epsilon_{z}=\left(F_{z z}\right)^{1 / 2}\right]$ as a function of $\sqrt{s}$. The numbers for the $F_{i j}$ are to be divided by $N$ and the number for the $\epsilon_{\gamma}, \epsilon_{z}$ by $\sqrt{N}$.

\begin{tabular}{lcccccccccccc}
\hline \hline$\sqrt{s}$ & 165 & 170 & 175 & 180 & 185 & 190 & 195 & 200 & 250 & 300 & 400 & 500 \\
\hline$F_{\gamma \gamma}$ & 91105 & 20060 & 10134 & 6500 & 4655 & 3560 & 2846 & 2349 & 813 & 625 & 1352 & 3960 \\
$F_{z z}$ & 33272 & 8709 & 4761 & 3211 & 2393 & 1894 & 1560 & 1323 & 538 & 421 & 871 & 2609 \\
$F_{\gamma z}$ & -54652 & -13141 & -6920 & -4556 & -3330 & -2591 & -2103 & -1760 & -659 & -511 & -1083 & -3112 \\
$\rho$ & -0.992 & -0.994 & -0.996 & -0.997 & -0.998 & -0.998 & -0.998 & -0.998 & -0.997 & -0.995 & -0.997 & -0.999 \\
$\epsilon_{\gamma}$ & 302 & 142 & 101 & 81 & 68 & 60 & 53 & 48 & 29 & 25 & 37 & 63 \\
$\epsilon_{z}$ & 182 & 93 & 69 & 57 & 49 & 44 & 39 & 36 & 23 & 21 & 30 & 51 \\
\hline \hline
\end{tabular}


TABLE IV. The dependence of the angle $\phi$ on $\sqrt{s}$.

\begin{tabular}{lcccccccc}
\hline \hline$\sqrt{s}(\mathrm{GeV})$ & 165 & 170 & 175 & 180 & 185 & 190 & 195 & 200 \\
$\phi(\mathrm{deg})$ & -31.1 & -33.1 & -34.3 & -35.1 & -35.7 & -36.2 & -36.3 & -36.9 \\
\hline \hline
\end{tabular}

$=161 \mathrm{GeV}$ to 0.73 at $\sqrt{s}=161 \mathrm{GeV}$. This energy dependence suggests that one could extract the two form factors $f_{3}^{\gamma}$ and $f_{3}^{Z}$ from $\sigma_{1}$ alone, by combining the experiments at different energies and by assuming that $f_{3}^{\gamma}$ and $f_{3}^{Z}$ are energy independent. This approach seems to be complementary to measuring $f_{3}^{\gamma}$ and $f_{3}^{Z}$ at each energy using the system of Eq. (3.27). One way of establishing this it to consider the covariant ellipses of constant likelihood in the two-dimensional space of $f_{3}^{\gamma}$ and $f_{3}^{Z}$, given by [37]

$$
1=\left(\frac{1}{1-\rho^{2}}\right)\left[\left(\frac{f_{3}^{\gamma}}{\epsilon_{\gamma}}\right)^{2}+\left(\frac{f_{3}^{Z}}{\epsilon_{z}}\right)^{2}-2 \rho\left(\frac{f_{3}^{\gamma}}{\epsilon_{\gamma}}\right)\left(\frac{f_{3}^{Z}}{\epsilon_{z}}\right)\right]
$$

or, equivalently,

$$
\left(f_{3}^{Z}\right)=\frac{\boldsymbol{\epsilon}_{z}}{\boldsymbol{\epsilon}_{\gamma}}\left\{\rho\left(f_{3}^{\gamma}\right) \pm\left(1-\rho^{2}\right)^{1 / 2}\left[\boldsymbol{\epsilon}_{\gamma}^{2}-\left(f_{3}^{\gamma}\right)^{2}\right]^{1 / 2}\right\}
$$

The ellipse is centered around $(0,0)$ because we have assumed for simplicity that the average values of $f_{3}^{\gamma}$ and $f_{3}^{Z}$ are zero, and is rather elongated due to the high value of $\rho$. Its principal axes make an angle $\phi$ relative to the coordinate system, given by

$$
\tan 2 \phi=\frac{2 \rho \epsilon_{\gamma} \epsilon_{z}}{\epsilon_{\gamma}^{2}-\epsilon_{z}^{2}}
$$

The dependence of the angle $\phi$ on $\sqrt{s}$ is shown in Table IV.

Evidently the dependence of $\phi$ on $\sqrt{s}$ is very mild; thus one expects to find (with $68.3 \%$ probability) the values of $f_{3}^{\gamma}$ and $f_{3}^{Z}$ in the interior of the (very slowly) rotating ellipse, regardless of the energy.

We hasten to emphasize that the above arguments are suggestive at best; a more detailed analysis using actual experimental data (and error analysis techniques) is needed in order to determine the feasibility of the proposed measurements, especially in the LEP2 context.

\section{CONCLUSIONS}

In this paper we have presented a set of sum rules relating the anomalous gauge boson couplings to the unpolarized differential cross section of the process $e^{+} e^{-} \rightarrow W^{+} W^{-}$. These sum rules involve only those anomalous couplings which separately conserve $C$ and $P$, and have been derived under the assumption that the produced $W$ bosons are strictly on shell. For this case we have defined four observables, called $\sigma_{i}, i=1,2,3,4$, which are linear combinations of the deviations of the trilinear gauge couplings from their SM values. The $\sigma_{i}$ observables can be extracted from the experimentally measured differential cross section by (i) subtracting out the known tree-level value of the differential cross section in the absence of anomalous couplings; (ii) multiplying the remainder by the angular dependence of the $t$-channel propagator; the latter is also an experimentally known quantity, since it only depends on the center-of-mass energy $s$, and scattering angle $\theta$; (iii) convoluting the resulting expressions with four appropriately constructed polynomials in $\cos \theta$ of maximum degree 3.

The role of these observables is twofold: On the one hand, two of these observables, namely $\sigma_{1}$ and $\sigma_{4}$, represent direct measurements of the magnetic moments $G_{M}^{\gamma}$ and $G_{M}^{Z}$ of the $W$, while the other two constitute model independent constraints (sum rules) between the remaining anomalous couplings. Thus, the two magnetic moments of the $W$ boson can be separately determined from the measurement of the unpolarized differential cross section. On the other hand, these observables are useful for testing dynamical models which predict sizable anomalous couplings. This is a direct consequence of the fact that some of those models predicts constraints between the anomalous couplings, which, in turn, can be directly translated to relations among the $\sigma_{i}$ observables. We have demonstrated this possibility in the context of a composite model, and a model based on an effective gauge-invariant Lagrangian.

Although we have restricted our discussion to couplings that respect $C$ and $P$, this method can be followed step by step also in the case where the trilinear vertex assumes its most general form. Of course, the system of equations that would correspond to Eq. (3.18) will be modified; in particular, it is not clear whether one would still be able to isolate $G_{M}^{\gamma}$ and $G_{M}^{Z}$, as happened in the simpler case we have considered here.

It would be interesting to determine how the analysis and results presented here are modified by the off-shellness effects of the $W$. This next step may be necessary in view of the fact that the cross section for on-shell $W$ pair production will not be measured with sufficient accuracy at LEP2. Such an analysis is complicated not only due to the large number of additional tree-level Feynman diagrams contributing to the process $e^{+} e^{-} \rightarrow W W \rightarrow 4 f$, but also by the fact that the (off-shell) W's may be resonant [2]. Calculations in this direction are already in progress.

\section{ACKNOWLEDGMENTS}

We thank C. G. Papadopoulos for various very useful and informative discussions, and E. Sanchez Alvaro and C. Palomares for communicating to us the interest of the L3 Collaboration in the present work. The work of J.P. was supported by Grant No. TMR-ERBFMBICT 972024. The work of K.P. was supported by a Marie-Curie TMR grant ERBFMBICT 961003. 
[1] F. Boudjema, Phys. Rev. D 36, 969 (1987); G. Belanger, F. Boudjema, and D. London, Phys. Rev. Lett. 65, 2943 (1990).

[2] Z. Ajaltouni et al., "Physics at LEP2,' Report No. CERN 96-01, 1996, Vol. 1, p. 525.

[3] F. Berends et al., J. Phys. G 24, 405 (1998).

[4] J. Ellison and J. Wudka, "Study of Trilinear Gauge Boson Couplings at the Tevatron Collider,' hep-ph/9804322, Ann. Rev. Nucl. Part. Sci. (to be published), and references therein.

[5] K. J. F. Gaemers and G. J. Gounaris, Z. Phys. C 1, 259 (1979).

[6] K. Hagiwara, R. D. Peccei, D. Zeppenfeld, and K. Hikasa, Nucl. Phys. B282, 253 (1987).

[7] U. Baur and D. Zeppenfeld, Nucl. Phys. B308, 127 (1988); B325, 253 (1989); E. N. Argyres et al., Phys. Lett. B 272, 431 (1991); E. N. Argyres et al., ibid. 280, 324 (1992); F. K. Diakonos et al., ibid. 303, 177 (1993).

[8] P. Mery, M. Perrottet, and F. M. Renard, Z. Phys. C 36, 249 (1987); 38, 579 (1988).

[9] G. Gounaris, J. Layssac, G. Moultaka, and F. M. Renard, Int. J. Mod. Phys. A 8, 3285 (1993).

[10] J. Papavassiliou, E. de Rafael, and N. J. Watson, Nucl. Phys. B503, 79 (1997).

[11] S. D. Drell and A. C. Hearn, Phys. Rev. Lett. 16, 908 (1966); S. B. Gerasimov, Yad. Fiz. 2, 598 (1965) [Sov. J. Nucl. Phys. 2, 430 (1966)]; S. J. Brodsky and S. D. Drell, Phys. Rev. D 22, 2236 (1980).

[12] S. J. Brodsky and J. R. Hiller, Phys. Rev. D 46, 2141 (1992).

[13] M. Kuroda, F. M. Renard, and D. Schildknecht, Phys. Lett. B 183, 366 (1987).

[14] A. De Rújula, M. B. Gavela, P. Hernandez, and E. Massó, Nucl. Phys. B384, 3 (1992).

[15] K. Hagiwara, S. Ishihara, R. Szalapski, and D. Zeppenfeld, Phys. Lett. B 283, 353 (1992).

[16] K. Hagiwara, S. Ishihara, R. Szalapski, and D. Zeppenfeld, Phys. Rev. D 48, 2182 (1993).

[17] G. Gounaris and F. M. Renard, Z. Phys. C 59, 133 (1993).

[18] W. A. Bardeen, R. Gastmans, and B. Lautrup, Nucl. Phys. B46, 319 (1972).

[19] Yu. L. Dokshitzer, D. I. Dyakonov, and S. I. Troyan, Phys. Rep. 58, 269 (1980); A. Andraši and J. C. Taylor, Nucl. Phys. B192, 283 (1981); D. M. Capper and G. Leibbrandt, Phys. Rev. D 25, 1002 (1982); G. Leibbrandt, Rev. Mod. Phys. 59, 1067 (1987).
[20] K. Fujikawa, B. W. Lee, and A. I. Sanda, Phys. Rev. D 6, 2923 (1972).

[21] E. N. Argyres, G. Katsilieris, A. B. Lahanas, C. G. Papadopoulos, and V. C. Spanos, Nucl. Phys. B391, 23 (1993).

[22] J. Papavassiliou and K. Philippides, Phys. Rev. D 48, 4255 (1993).

[23] A. B. Lahanas and V. C. Spanos, Phys. Lett. B 334, 378 (1994); hep-ph/9504340; E. N. Argyres, A. B. Lahanas, C. G. Papadopoulos, and V. C. Spanos, Phys. Lett. B 383, 63 (1996); A. Arhrib, J. L. Kneur, and G. Moultaka, ibid. 376, 127 (1996).

[24] X. G. He, J. P. Ma, and B. H. J. McKellar, Phys. Lett. B 304, 285 (1993); C. P. Burgess and A. Pilaftsis, ibid. 333, 427 (1994).

[25] L. F. Abbott and E. Fahri, Phys. Lett. 101B, 69 (1981); Nucl. Phys. B189, 547 (1981); M. Claudson, E. Fahri, and R. L. Jaffe, Phys. Rev. D 34, 873 (1986).

[26] J. M. Cornwall, D. N. Levin, and G. Tiktopoulos, Phys. Rev. Lett. 30, 1268 (1973); 31, 573(E) (1973); Phys. Rev. D 10, 1145 (1974).

[27] C. H. Llewellyn Smith, Phys. Lett. 46B, 233 (1973).

[28] U. Baur and D. Zeppenfeld, Phys. Lett. B 201, 383 (1988).

[29] W. Alles, Ch. Boyer, and A. J. Buras, Nucl. Phys. B119, 125 (1977).

[30] J. Maalampi, D. Schildknecht, and K. H. Schwarzer, Phys. Lett. 166B, 361 (1986).

[31] G. J. Gounaris, J. Layssac, and F. M. Renard, Phys. Lett. B 332, 146 (1994); G. J. Gounaris, J. Layssac, J. E. Paschalis, and F. M. Renard, Z. Phys. C 66, 619 (1995); G. J. Gounaris, F. M. Renard, and G. Tsirigoti, Phys. Lett. B 338, 51 (1994); 350, 212 (1995).

[32] A. Manohar and H. Georgi, Nucl. Phys. B234, 189 (1984).

[33] L3 Collaboration, E. Sanchez Alvaro and C. Palomares (private communication).

[34] R. L. Sekulin, Phys. Lett. B 338, 369 (1994).

[35] C. G. Papadopoulos, Phys. Lett. B 386, 442 (1996).

[36] W. T. Eadie et al., Statistical Methods in Experimental Physics (North-Holland, Amsterdam, 1971).

[37] See, for example, A. G. Frodesen, O. Skjeggestad, and H. Tofte, Probability and Statistics in Particle Physics (Columbia University Press, New York, 1979). 